\title{
BMJ open The value of intervening for intimate partner violence in South African primary care: project evaluation
}

\author{
Kate Joyner, ${ }^{1}$ Robert James Mash ${ }^{2}$
}

To cite: Joyner K, Mash RJ. The value of intervening for intimate partner violence in South African primary care: project evaluation. BMJ Open 2011:1:e000254. doi:10. 1136/bmjopen-2011-000254

- Prepublication history for this paper is available online. Additional supplementary appendices are published online only. To view these files please visit the journal online (http://bmjopen.bmj. com/content/early/recent).

Received 15 July 2011 Accepted 30 September 2011

This final article is available for use under the terms of the Creative Commons Attribution Non-Commercial 2.0 Licence; see http://bmjopen.bmj.com

${ }^{1}$ Stellenbosch University, Division of Nursing, Cape Town, South Africa

${ }^{2}$ Stellenbosch University, Division of Family Medicine and Primary Care, Cape

Town, South Africa

Correspondence to

Dr Kate Joyner;

kjoy@sun.ac.za

\section{ABSTRACT}

Objectives: Intimate partner violence (IPV) is an important contributor to the burden of disease in South Africa. Evidence-based approaches to IPV in primary care are lacking. This study evaluated a project that implemented a South African protocol for screening and managing IPV. This article reports primarily on the benefits of this intervention from the perspective of women IPV survivors.

Design: This was a project evaluation involving two urban and three rural primary care facilities. Over 4-8 weeks primary care providers screened adult women for a history of IPV within the previous 24 months and offered referral to the study nurse. The study nurse assessed and managed the women according to the protocol. Researchers interviewed the participants 1 month later to ascertain adherence to their care plan and their views on the intervention.

Results: In total, 168 women were assisted and 124 (73.8\%) returned for follow-up. Emotional (139, $82.7 \%)$, physical $(115,68.5 \%)$, sexual $(72,42.9 \%)$ and financial abuse $(72,42.9 \%)$ was common and 114 $(67.9 \%)$ were at high/severe risk of harm. Adherence to the management plan ranged from testing for syphilis $10 / 25(40.0 \%)$ to consulting a psychiatric nurse $28 / 58(48.3 \%)$ to obtaining a protection order $28 / 28(100.0 \%)$. Over $75 \%$ perceived all aspects of their care as helpful, except for legal advice from a non-profit organisation. Women reported significant benefits to their mental health, reduced alcohol abuse, improved relationships, increased self-efficacy and reduced abusive behaviour. Two characteristics seemed particularly important: the style of interaction with the nurse and the comprehensive nature of the assessment.

Conclusion: Female IPV survivors in primary care experience benefit from an empathic, comprehensive approach to assessing and assisting with the clinical, mental, social and legal aspects. Primary care managers should find ways to integrate this into primary care services and evaluate it further.

Interpersonal violence is the second highest contributor to the burden of disease after HIV and AIDS, with intimate partner violence (IPV) accounting for $62.4 \%$ of the

\section{ARTICLE SUMMARY}

Article focus

- Did women experiencing IPV find assessment and management in primary care beneficial?

- What aspects of their care plan did they adhere to?

- What aspects of their care plan did they find most helpful?

Key messages

- Women diagnosed with IPV in primary care perceive benefit from an intervention characterised by both empathic, non-judgemental and a comprehensive approach to the clinical, mental, social and legal aspects.

- Women reported benefits to their mental health, alcohol use, relationships and experience of abusive behaviour.

- IPV survivors were most proactive about securing protection orders, laying criminal charges and testing for pregnancy post-intervention.

Strengths and limitations of this study

- A comprehensive biopsychosocial and forensic intervention in primary care was tested, securing follow-up that revealed its value.

- The study was conducted under usual working conditions and resource availability making the findings applicable to the primary care context.

- Although the study only involved five purposely selected facilities, the rural/urban mix makes it likely that these are fairly typical.

- Obsequiousness bias was reduced by different researchers conducting the follow-up interviews.

- Follow-up after 1 month is too short to predict the longer-term consequences of the intervention.

- The study measured the effect of the intervention on the abuse indirectly via participant selfreports.

total burden in women. ${ }^{1}$ South Africa's intimate femicide rate is the highest globally. ${ }^{2}$ IPV appears to be twice as common in rural South African settings compared with urban, with a complex interplay of racial, gender and economic forces, maintaining women in 
abusive situations. ${ }^{3}$ The use of violence to maintain dominance in interpersonal relationships forms a crosscultural cornerstone of masculinity in South Africa. ${ }^{4}$ For these reasons, it is crucial that healthcare providers attend to IPV survivors' needs comprehensively.

Gender-based violence is an umbrella term for forms of interpersonal violence characterised by gendered power imbalances. Fitting within this, IPV refers to emotional, physical, sexual or financial abuse between intimates. It also fits within broader subcategories of gender-based violence, namely family and domestic violence.

South African laws and policy on violence against women acknowledge it to be a significant human rights and public health issue, yet extreme levels of genderbased violence, poverty and HIV infection among women reveal a chasm between the daily lives of female citizens and apparent gains in the public sphere. ${ }^{5}$ South Africa's Domestic Violence Act (116 of 1998) includes a definition of domestic violence so comprehensive it is a legislative standard bearer internationally. ${ }^{6}$ An inexpensive and unsophisticated civil procedure for obtaining a protection order clearly demarcates the duties of the police. ${ }^{7}$ Unfortunately, specific responsibilities for healthcare providers are not outlined and a gulf exists between the contents of the act and its practical implementation.

The WHO now positions IPV as an urgent public health priority. ${ }^{8}$ Internationally, evidence-based interventions in primary care settings are lacking. ${ }^{9} 10 \mathrm{~A}$ systematic review on screening for IPV in primary care concludes that while 'domestic violence is a common problem with major health consequences for women, implementation of screening programmes cannot be justified'. ${ }^{11}$ They call for evidence of the benefit of specific interventions and lack of harm from screening.

Paradoxically, universal screening for IPV in healthcare settings is commonly endorsed by international guidelines. ${ }^{12}$ However, in poorly resourced contexts such as South Africa the use of case finding based on the presentation of specific signs or symptoms of abuse is recommended. ${ }^{13}$ This focuses healthcare resources and providers' time on those needing immediate healthcare.

In South African primary care, case finding is not practised routinely and IPV remains hidden beneath other diagnoses. Health providers are mostly reluctant to ask about IPV and patients rarely disclose the problem spontaneously. Providers are also unsure how to manage IPV as it has complex clinical, mental, social and legal implications. They tend not to accept that IPV is a health problem, and in a system already overburdened by other challenges, may be unwilling to legitimise IPV as part of their work. ${ }^{14}$

Evaluations of initiatives addressing IPV are lacking, and frequently focus on the criminal justice system. ${ }^{15}$ However, lack of evidence should not be equated with lack of effectiveness. ${ }^{16}$ Enquiry about IPV offers a way of 'uncovering and reframing a hidden stigma' to provide benefit, even if no action follows forthwith. ${ }^{17}$

This article presents part of a larger study that implemented and evaluated a South African protocol for screening and managing women living with IPV in local primary healthcare settings. ${ }^{18} 14$ This article reports on how acceptable this protocol was to the women and what they perceived as its benefits.

\section{METHODS}

\section{Study design}

The overall design for the larger study was action research. The action or project was the implementation of a protocol for the screening and management of IPV in primary care. The research, by a cooperative enquiry group, evaluated the experience of the primary care staff and women survivors of IPV and reflected on their own experience of using the protocol. This article presents quantitative and qualitative data that were collected 1 month after the initial screening and use of the protocol and which was designed to obtain feedback from the women on their experience and response to the intervention. For the purposes of this article we have described this as project evaluation.

\section{Study setting}

Two urban and three rural healthcare facilities were purposefully selected in the Western Cape. Selection was based on their willingness to participate, availability of a psychiatric nurse and a private space for the study nurse. Otherwise facilities were typical of primary care in the Western Cape. Patients in ambulatory primary care were usually seen by a nurse practitioner.

The two urban community health centres were situated in formerly designated 'black' and 'coloured' townships in Cape Town. One rural site was a community health centre in a large rural town and in a former 'coloured group area'. The other two rural sites were remote, smaller clinics in farming communities. All these public sector facilities served low socioeconomic groups who were not medically insured.

\section{Ethical considerations}

Guided by the ethical considerations and research solutions recommended to the WHO by the International Research Network on Violence Against Women, ${ }^{19}$ the following issues were prioritised:

- The safety of respondents and the research team was paramount and infused all project decisions.

- Study nurses received specialised training and ongoing support. During the research process regular debriefing enabled the study nurses to discuss the challenges and feelings raised. Consultations with a psychologist were also made available if wanted. This accorded with the ethical responsibility to reduce work stress and avert negative consequences. 
- Interviews were conducted privately and confidentially by members of the research team, and anonymity was ensured.

- Participants provided informed, written consent and could withdraw or reschedule at their will.

- Ethics approval was given by the Health Research Ethics Committee at Stellenbosch University (reference no $6 / 10 / 216)$.

\section{Selection of women}

As part of a larger study, all primary care providers at the facilities were asked to screen all female patients, of 18 years and older, for a history of IPV during the previous 24 months. Facility managers agreed to screen women for 4-8 weeks. However many providers were resistant and uncooperative with our request for universal screening.

In-service training of the healthcare practitioners was given by the researchers and they were provided with a prompt tool that listed the questions, such as, "How are things going in your relationship?" or "In this clinic we ask all women patients if they have ever experienced any form of abuse. Have you ever experienced abuse by your partner?" Women identified as having experienced IPV were offered a referral to the study nurse.

\section{Intervention}

The authors recruited and trained two nurse practitioners to provide the intervention. These study nurses, including the principal author, then obtained consent and built rapport with the women before proceeding with the comprehensive assessment and management plan described below (see supplementary appendix A, available online only). The protocol tested in this study was developed in Cape Town by the Consortium on Violence Against Women with the help of Canadian specialists in IPV. ${ }^{18}$ The suggested assessment and management plan included the following:

- A systematic history of the type and frequency of abuse specifying particular aspects of emotional, sexual, financial and physical abuse.

- A comprehensive medicolegal history including previous HIV tests, sexually transmitted infections, risk of pregnancy, previous attempts to enlist assistance from the police or law courts and forensic documentation of injuries. If necessary, the woman was offered further testing or treatment.

- The original protocol neglected aspects of mental health. Nine questions to screen for mental health problems were added from a locally adapted version of the WHO's toolkit for the recognition, diagnosis and treatment of mental disorders in primary care. $^{20}$ Each question was designed to increase the likelihood of either depression, anxiety disorders, post-traumatic stress disorder (PTSD) or substance abuse. If as a result of this screening tool a mental problem was suspected then the participant was referred to the psychiatric nurse for a full assessment.
- A safety assessment tool intended to ascertain the woman's risk of serious injury. Discussion of a safety plan then followed with referral to other health, social, legal or police services.

The safety assessment tool used in the protocol appeared to have been formulated on the assumption that women would present with a serious injury, that her abuser would be living with her and that he might kill her if/when she gets home, or soon thereafter. The predictive value of the assessment needs to be further evaluated in relation to a specific outcome such as the frequency of abuse or the likelihood of being killed in the immediate future. How accurately the tool represents the most important risk factors in the South African setting needs further study. All risk factor counted equally, for example, "Has he threatened to kill you?" scores the same as "Is he presently at home?", and yet factors may have different weighting in terms of the risk that they represent.

Before leaving, women were given an appointment for a follow-up interview 1 month later.

\section{Data collection}

Data were extracted from the record of the initial consultation to give a profile of the management plans. The intended management plan was available to the researcher at the follow-up interview.

A different researcher conducted the follow-up interviews. A semi-structured questionnaire explored adherence to, and perceptions of, the care planned during the initial consultation (see supplementary appendix B, available online only). Participants reflected on how useful this had been, how they felt about their IPV situation now, and whether they had acted upon their intended plans. These data are based on the detailed notes of the researcher who recorded participants' responses to the open questions. All data were collected in the same way. Sometimes narratives were recorded verbatim in the first person and sometimes paraphrased in the third person.

\section{Data analysis}

The quantitative data were analysed by Stellenbosch University's Centre for Statistical Consultation in terms of simple frequency tables. The qualitative data were coded and analysed according to the framework method to understand how participants experienced the intervention. ${ }^{21}$

\section{FINDINGS}

\section{Profile of the women}

Participants comprised 168 women with 56 (33.3\%) from urban and $112(66.6 \%)$ from rural facilities. Overall, the mean age was 36.7 years and there was no significant difference between those who did (36.9 years) and did not (36.0 years) attend follow-up $(\mathrm{p}=0.59)$. Women were mostly married $(82,48.8 \%)$, cohabiting $(36,21.4 \%)$ or currently single $(35,20.8 \%)$, and had a mean of 2.5 children (range $0-6$ children). 
Table 1 describes the frequency and range of abuse. Over the 2 years preceding this study, 96 (57\%) participants admitted to being abused more than 20 times, 27 (16\%) between 11 and 20 times and 45 (27\%) had been abused 10 or fewer times. Each woman experienced on average eight forms of abuse over extended periods. Emotional abuse was commonest and ranged from verbal abuse to restricted contact with family and friends, to control of the woman's freedom to pursue activities. Physical abuse came a close second, with 115 (68\%) having experienced beatings, $82(50 \%)$ kicked, and alarmingly $55(34 \%)$ had been choked. There was no difference in the types of abuse between the women who did and did not attend follow-up.

Table 2 describes their degree of risk according to the safety scores, and shows that $114(67.9 \%)$ were at high or severe risk. The fact that $77(45.8 \%)$ believed he was capable of killing indicates the atmosphere of fear pervading the households. Apart from the danger to the woman herself, $91(54.2 \%)$ admitted that their partners had threatened to kill their children. Overall, the mean safety assessment score was 4.85 and there was also no significant difference between those who did (4.81) and did not (4.96) attend follow-up $(\mathrm{p}=0.75)$.

Table 3 shows the high levels of suspected mental problems among the women and how many were referred for further assessment. More than two-thirds of women were suspected of having depression or anxiety disorders, and a third were specifically suspected of
PTSD. A quarter of women were suspected to have problems with substance abuse, most commonly alcohol. There was no difference in the mental health profile of the women who did and did not attend for follow-up.

Impact and value of different aspects of the intervention Out of the 168 women $124(73.8 \%)$ returned for the follow-up interview. Table 4 shows key elements of the management plan and the extent to which women acted on these plans. Significantly, 28/28 (100\%) of women followed through on applying for a protection order, 16/ $19(84.2 \%)$ proceeded to lay a charge and 23/24 $(95.8 \%)$ saw the social worker.

Table 5 shows the perceived usefulness of different aspects of the intervention. Over $75 \%$ of women found counselling from a non-profit organisation (NPO), safety assessment and planning, referral to the psychiatric nurse, applying for a protection order and laying a criminal charge useful, when it was part of their management plan.

\section{Therapeutic effect of empathic listening}

Participants explained that telling their story and feeling understood was in itself beneficial.

“... found the intervention interesting ... felt that ... she could talk to someone and they actually heard her. She also felt that she could trust the study nurse. She described the social worker and psychiatric nurse and how they just said, 'yes, yes' and never really understand.

Table 1 Prevalence of different types of abuse

\begin{tabular}{|c|c|c|c|c|}
\hline Abuse behaviour & All $N=168, n(\%)$ & $\begin{array}{l}\text { Follow-up } \\
N=124, \mathrm{n}(\%)\end{array}$ & $\begin{array}{l}\text { No follow-up } \\
N=44, n(\%)\end{array}$ & p Value \\
\hline \multicolumn{5}{|l|}{ Emotional } \\
\hline Shouting & 139 (82.7) & 105 (84.7) & $34(77.3)$ & 0.27 \\
\hline Name calling & $136(81.0)$ & 102 (82.3) & $34(77.3)$ & 0.47 \\
\hline Threatening & $94(56.0)$ & $69(55.6)$ & $25(56.8)$ & 0.89 \\
\hline Restricting contact & $84(50.0)$ & $64(51.6)$ & $20(45.4)$ & 0.48 \\
\hline Controlling activities & $63(37.5)$ & 47 (37.9) & $16(36.4)$ & 0.86 \\
\hline Accusations & 19 (11.3) & $17(13.7)$ & $2(4.5)$ & 0.07 \\
\hline \multicolumn{5}{|l|}{ Physical } \\
\hline Hit & $115(68.5)$ & $86(69.3)$ & 29 (65.9) & 0.67 \\
\hline Pushed & 90 (53.6) & $56(45.2)$ & $26(59.1)$ & 0.11 \\
\hline Kicked & $82(48.8)$ & $46(37.1)$ & 23 (52.3) & 0.08 \\
\hline Use of weapon & 69 (41.1) & $65(52.4)$ & $25(56.8)$ & 0.61 \\
\hline Choked & 57 (33.9) & 41 (33.1) & $16(36.4)$ & 0.69 \\
\hline Burnt & $8(4.8)$ & $5(4.0)$ & $3(6.8)$ & 0.47 \\
\hline Hair pulled & $2(1.2)$ & $2(1.6)$ & $0(0.0)$ & 0.27 \\
\hline \multicolumn{5}{|l|}{ Sexual } \\
\hline Infidelity & 72 (42.9) & $53(42.7)$ & $19(43.2)$ & 0.96 \\
\hline Sexual coercion & $55(32.7)$ & 42 (33.9) & $13(29.5)$ & 0.60 \\
\hline Unwanted touching & $41(24.4)$ & $34(27.4)$ & 7 (15.9) & 0.11 \\
\hline STI & $41(24.4)$ & $32(25.8)$ & $9(20.4)$ & 0.47 \\
\hline \multicolumn{5}{|l|}{ Financial } \\
\hline Withholding money & 72 (42.9) & $55(44.3)$ & $17(38.6)$ & 0.51 \\
\hline Controlling decisions & 25 (14.9) & 19 (15.3) & $6(13.6)$ & 0.78 \\
\hline Taking money & $23(13.7)$ & $19(15.3)$ & $4(9.1)$ & 0.28 \\
\hline
\end{tabular}




\begin{tabular}{lcr} 
Table 2 Safety assessment score & & \\
\hline & $\mathbf{n}=168$ & $\%$ \\
\hline Safety assessment score & 3 & 1.8 \\
Not done & 50 & 29.8 \\
Caution (0-3) & 87 & 51.8 \\
High risk (4-7) & 27 & 16.1 \\
Severe risk (8-11) & & \\
Safety assessment fields & 125 & 74.4 \\
Threatened with physical violence? & 109 & 64.9 \\
Threatened children with violence? & 102 & 60.7 \\
Firearm in house? & 92 & 54.8 \\
Made a death threat? & 91 & 54.2 \\
Threatened to kill children? & 77 & 45.8 \\
Capable of killing? & 62 & 36.9 \\
Substance use before last abuse? & 54 & 32.1 \\
Police intervention necessary? & 41 & 24.4 \\
Is he in the house? & 35 & 20.8 \\
Has abuse escalated in severity? & 33 & 19.6 \\
Received medical treatment for injuries? & &
\end{tabular}

The study nurse gave her advice and told her what she could do." (036)

Being able to be honest in a supportive and nonjudgemental interaction was helpful:

"No one had ever spoken to her openly before and it was the first time she was able to be completely honest about everything. She does not want to give up on her marriage and feels it would be best if she and her husband received counselling together." (122)

The style of interaction was different from usual and facilitated honest reflection on the situation:

"Doctor had asked her before why she was so stressed but she was too embarrassed to tell him. But she was able to talk to (the study nurse) and felt it was good to have evaluated her situation." (154)

\section{Value of clinical care}

Participants appreciated having their overall health as women taken seriously, particularly as many aspects were examined, which were not usually dealt with. Many felt relieved to be tested for HIV or assessed for sexually transmitted infections. Adherence to other ongoing clinical care, such as antiretroviral treatment, appeared also to improve:
"When patient first came she describes herself as tense and hysterical. While talking to the study nurse she experienced a change inside ... hope .... Before she met her she never thought she could reach the place she is now ... (decreased CD4 count, attending ARV clinic, has gained weight).” (048)

\section{Safety assessment and planning}

The safety assessment was found to be useful for the following range of reasons:

“... made her feel better after hearing that it was wrong to be forced to have sex" (149)

"... opened her eyes as she learnt that he should not hurt her like that" (138)

"... saw how dangerous her situation was and decided to get help (a protection order)" (097)

"... made her aware of her own faults as well" (082)

Participants cited many reasons why they found the safety plan useful:

\begin{abstract}
"She is very afraid as feels he could kill her. Good to discuss what to do and make a plan - not tell him anything. Just leave." (156)

"In the past always had to scramble around and look for things in an emergency but now all her things are in one place." (123)
\end{abstract}

\section{Referral to other resources}

Knowing about the available resources and being offered referral was appreciated:

"The intervention helped a lot. She did not know that there were so many people to help her. She had been to social workers before, but it is not the same. She now does not feel alone anymore." (025)

The referral letter had helped to access the services required:

“...before ' $x$ ' court had refused to help her, but with the letter they gave her a protection order. Now she feels happy." (053)

Many participants expressed relief at discovering they have rights, and where to find help when they need it. Many expressed that the questions and information opened up a new world, linking them to resources and showing ways to change their circumstances.

Table 3 Referred for assessment of mental health problems

\begin{tabular}{lrlll}
$\begin{array}{l}\text { Referred for further } \\
\text { assessment for }\end{array}$ & All N=168, $\mathbf{n}(\%)$ & Follow-up $\mathbf{N = 1 2 4 , ~} \mathbf{n}(\%)$ & No follow-up N=44, $\mathbf{n}(\%)$ & $\mathbf{p ~ V a l u e ~}$ \\
\hline Depression & $110(65.5)$ & $79(64.7)$ & $31(72.1)$ & 0.37 \\
PTSD & $65(38.7)$ & $44(35.5)$ & $21(48.8)$ & $30(69.8)$ \\
Anxiety & $121(72.0)$ & $91(74.6)$ & $11(25.6)$ & 0.12 \\
Substance abuse & $42(25.0)$ & $31(25.0)$ & &
\end{tabular}


Table 4 Adherence of women to plans made by 1-month follow-up ( $N=124)$

\begin{tabular}{|c|c|c|c|c|}
\hline & \multicolumn{2}{|c|}{ Management plan } & \multirow{2}{*}{$\begin{array}{l}\text { Action } \\
\text { n (\%) }\end{array}$} & \multirow{2}{*}{$\begin{array}{l}\text { Adherence } \\
\mathrm{n} / \mathrm{N} \%\end{array}$} \\
\hline & $\mathbf{N}$ & $\%$ & & \\
\hline Wants HIV test & 56 & 45.2 & $31(25.0)$ & 55.4 \\
\hline Wants rapid plasma reagin test & 25 & 20.2 & $10(8.1)$ & 40.0 \\
\hline Wants pregnancy test & 6 & 4.8 & $10(8.1)$ & 100.0 \\
\hline Intends to obtain a protection order & 28 & 22.6 & $28(22.6)$ & 100.0 \\
\hline Intends to lay a charge & 19 & 15.3 & $16(12.9)$ & 84.2 \\
\hline Referral to NPO for legal support & 15 & 12.1 & $7(5.6)$ & 46.7 \\
\hline Referral to NPO counselling & 32 & 25.8 & $12(9.7)$ & 37.5 \\
\hline Referral to psychiatric nurse & 58 & 46.8 & $28(22.6)$ & 48.3 \\
\hline Referral to social worker & 24 & 19.4 & $23(18.5)$ & 95.8 \\
\hline
\end{tabular}

\section{Problems with police}

Most complaints referred to unsympathetic, impersonal, indiscreet or corrupt police. One participant's partner had paid the police off while another's recurrently abusive behaviour continued despite her protection order. Another explained when she had applied for a protection order she was not given a copy of the temporary order. When she laid a charge, she received a case number, but no copy of the statement or medical report. This failure of authorities to provide copies was commonly reported by participants.

\section{Negative reactions to the intervention}

Digging up the past had upset a participant. Another complained that she felt very frightened after the interview. One said that things were worse at home because following the intervention she informed her husband that she intended to divorce him and he threatened to take the children and jump under a train.

\section{Suggestions to improve the help offered}

A strong theme was the need for a support group:

"She would like to talk to other women who have been through the same situations. She would like to make friends and learn from other people." (083)

A professional rural woman desired referral to services outside her community as she felt too exposed to access services within it. Another would only see a female counsellor. Some wanted their partner to be 'spoken to' or couple counselling to prevent the impression that only women need help. Some resented a perceived onus on women to resolve problems rather than zero tolerance for men's abusive behaviour and proactive efforts to get functional interventions in place. Other participants requested practical assistance such as written advice or help with completing a protection order application. One woman expressed a need for solitude and space to reflect.

\section{Improved mental state}

At least 35 of the participants reported an improved mental state in terms of their mood, sociability or sense of wellbeing as well as decreased anxiety, suicidal ideation and alcohol abuse. Enhanced parenting emerged frequently as a theme.

\footnotetext{
"I used to feel sad all the time. I used to feel useless but now I feel responsible. I can take care of my children now. When I was drinking every day I would take them to a woman to look after them. Now I enjoy my kids and my life more ... since I went to press charges we have not been together and he has not hurt me either." (047)

"I was a wreck, but when I left her office I could face the world squarely again. She taught me things about myself I did not know. My problems were not solved then and there but I can now notice what I did not before and I feel better about myself as a person." (166)
}

Table 5 Perceived usefulness of different aspects of the intervention

\begin{tabular}{lcccc}
\hline Intervention & $\mathbf{N}$ & $\begin{array}{l}\text { Very helpful/helpful } \\
\mathbf{n}(\%)\end{array}$ & $\begin{array}{l}\text { Don't know } \\
\mathbf{n}(\%)\end{array}$ & $\begin{array}{l}\text { Unhelpful } \\
\mathbf{n}(\%)\end{array}$ \\
\hline Going to NPO for legal advice & 7 & $4(57.2)$ & $2(28.6)$ & $1(14.3)$ \\
Going to social worker & 23 & $16(69.6)$ & $4(17.4)$ & $3(13)$ \\
Going to NPO for counselling & 12 & $9(75.0)$ & $16(15.7)$ & $1(8.3)$ \\
Safety plan & 102 & $77(75.5)$ & $15(14.2)$ & $9(8.8)$ \\
Safety assessment & 106 & $85(80.1)$ & $0(3.6)$ & $4(5.7)$ \\
Protection order & 28 & $23(82.1$ & $0(0)$ & $2(7.1)$ \\
Going to psychiatric nurse & 28 & $26(92.8)$ & & $1(6.3)$ \\
Laying criminal charge & 16 & $15(93.8)$ & &
\end{tabular}




\section{Reduced isolation}

Some participants found that the intervention encouraged them to reduce their isolation by reconnecting with trustworthy friends or family members in a more open and honest way:

"Communication ... has improved. It is easier for her to disagree and she feels more independent, like she could manage without him. She had isolated herself quite a bit, but is now starting to reach out to her friends again." (026)

\section{More aware of their rights}

Women described no longer 'feeling lost' since they learnt about potentially helpful resources:

"For the first time in two years, she phoned the police and used her protection order. She appeared in front of the magistrate and since then he has lived with his family." (021)

Many discussed a notable improvement in the partners' behaviour in response to a letter the researcher had written to the police and/or magistrates' court. In most cases when the criminal justice system was effectively engaged the abusive behaviour stopped completely, usually due to fear of jail.

\section{Taking action}

On follow-up, some reported having taken action to enhance the quality of their lives. Eleven women ended the relationship after the intervention and reported at follow-up how relieved they felt:

“... after interview she got a protection order and ended her relationship with her gangster boyfriend who uses tic" (metamphetamine). (104)

\section{Improved relationships}

Many participants described an improvement in their family relationships:

"I went to a support group for abused women. A lot changed inside myself, like now I know how to talk to my husband. I was always angry and shouting at him, now we communicate better. I am much kinder to the kids. I feel happy now. Before I felt sad, wanted to cry and stay alone." (016)

\section{Change in own behaviour}

Many participants described how the intervention had motivated a change in their own behaviour. Reduced alcohol abuse was a particularly frequent effect, with enhanced parenting an attendant theme:

"Our relationship is going much better than before. I discussed certain things with him and he understood me. I am drinking much less than before and going to evening songs again. I never wanted to work on Saturdays but now I am." (164)

"It made her realise that she can no longer continue to use alcohol because it's detrimental to her baby. She feels she can talk about things easier and that she came out a stronger person, able to take a stand." (166)

"Yes, I am different inside. When he tries to provoke me with verbal abuse when drunk, I don't allow him to make me angry. He doesn't even listen to me, but I take me seriously." (007)

\section{Change in partners' behaviour}

Change in partners' behaviour was also reported and 56 reported an improved relationship with their partner:

\begin{abstract}
"There has been great change in her life. The intervention was very useful because the results have made her feel very happy. The situation at home has improved very much. Her husband has even been working in their garden and helping the children with homework - never before." (065)

"She found the whole experience useful because she got a protection order and there is now someone to stop him. Even though he was famous for his actions locally and people were aware of his behaviour, nothing was ever done. The protection order however has changed his behaviour." (078)
\end{abstract}

\section{No real change}

Thirty-nine participants reported that there had been no real change. The intractable challenges that pervaded these responses often involved alcoholism in either or both partners, financial constraints, HIV infection as well as participants' inertia to follow-up on referrals made. Even so, some reported that they had found the process useful:

\section{"No real change - he continues to withhold pay while expecting money from her with which to buy alcohol. Locked her and kids out of home while he was away drinking, very abusive on return. She wants a divorce." (133)}

A common refrain was, "When he is not drunk, he is such a nice guy."

Another trap was expressed as follows:

\begin{abstract}
"She needs the money that he provides ... a protection order will make him angry and he will not be able to work if he is in jail. She is still very scared of him but he gets angry if she addresses his behaviour. He must always be right." (156)
\end{abstract}

\section{DISCUSSION}

Women were simultaneously experiencing multiple types of abuse and were mostly at high or severe risk of injury and death. Women showed strong adherence to the components of their care plan, particularly applying for a protection order, laying a charge, referral to a social 
worker and obtaining a pregnancy test. Women described all aspects of the intervention as helpful, particularly counselling from a NPO, the safety assessment and planning, applying for a protection order, laying a criminal charge and seeing the psychiatric nurse.

Overall, $11(9 \%)$ women reported that they had ended their relationship, $56(45 \%)$ that their relationship had improved and $39(31 \%)$ that there was no change. These findings are congruent with previous work that suggests that most IPV survivors want the violence, not the relationship, to end. ${ }^{22}$ Women reported significant benefits to their mental health, reduced alcohol abuse, increased self-efficacy and reduced abusive behaviour.

Two characteristics of the intervention appeared particularly important: the style of interaction with the nurse and the comprehensive nature of the assessment.

Being encouraged to tell their story and being listened to with genuine interest and care emerged as key features of the interview that participants valued. This echoes other studies that speak of IPV survivors' need for compassionate, uninterrupted listening. ${ }^{23}{ }^{24}$ Clearly indicating to patients that violence is not a taboo topic is in itself a vital therapeutic component of any intervention. ${ }^{25}$ The safety assessment provided feedback on how the situation is perceived relative to a different norm, thus enhancing the discrepancy between 'normalcy' and their current situation. Our findings are congruent with other studies that emphasise the value of empathic, nonjudgemental interaction. ${ }^{26-28}$

This study addresses a key gap in the literature because it provides evidence of the benefits of a comprehensive intervention for IPV. ${ }^{9-11}$ In order to be comprehensive, assessment and care should include clinical, psychological, social, legal and advocacy components. Clinical aspects include preventing unwanted pregnancy, sexually transmitted infections and HIV testing. Psychological refers to the identification and treatment of potential mental health problems. Social encompasses referral to support groups, social workers and safety planning. Legal includes referral for a protection order.

Advocacy and referral to relevant resources has the potential to interrupt and prevent recurrent IPV and associated trauma while enhancing IPV survivors' quality of life. ${ }^{9}$ Six months of advocacy has been found to improve emotional health, personal safety, healthcare and the ability to get medication or treatment while decreasing unhealthy coping behaviours. ${ }^{29}$

\section{Strengths and limitations}

The hidden nature of IPV and the study design make it impossible to determine what proportion of women experiencing IPV in these communities attended the primary care facilities. It is also not possible to determine what proportion of women who did attend were identified. Fewer women than expected were identified and referred to the study, and the issues related to screening are presented elsewhere. ${ }^{13}$ It is possible that those women who disclosed were experiencing more severe forms of IPV and therefore were more likely to seek help.

The mixed methods facilitated in-depth understanding of how women experienced the intervention. Follow-up was, however, limited to 1 month so the study cannot determine if changes were sustained or if additional changes occurred subsequently. A longitudinal evaluation measuring the effect of the intervention on the abuse could provide further evidence of effectiveness.

\section{Implications and recommendation}

The study provides evidence that the intervention was perceived as useful by most participants. Implementation in primary care will require attention to enabling an empathic communication style and a comprehensive assessment and care plan. A proposed model of how the intervention can be integrated into local primary care is described elsewhere. ${ }^{30}$

The evaluation of this protocol has provided sufficient evidence to the Department of Health to pilot the model in the Western Cape. While this intervention focuses on women, future research should include working with men. As the model is implemented further research should monitor the identification of women via case finding in primary care compared with the expected prevalence in the community served, explore which women disclose to and take up services initiated by health professionals and evaluate benefits in the longer term. It may also be useful to relate the introduction of the model to outcomes such as mortality from interpersonal violence among women at the sub-district level.

The communication style mirrors that of motivational interviewing, which helps people make difficult decisions about behaviour change. ${ }^{31}$ Characterised by empathy, clear direction, collaboration, evocation of the client's perspective and solutions, its approach respects autonomy. Training in motivational interviewing as well as the conceptualisation of IPV as a chronic rather than an acute condition should be considered in the roll-out of this model.

\section{CONCLUSION}

Women diagnosed with IPV in primary care perceive clear benefit, at least in the short term, from an intervention characterised by both empathic, non-judgemental listening and a comprehensive approach to assessing and managing the clinical, mental, social and legal aspects. District managers should integrate this approach into primary care services and evaluate it further.

Acknowledgements Thanks to all the IPV survivors, health providers, health system managers, research assistants and referral resources who participated in this study, thereby making it possible. Thanks also to Kees van der Waal for his insight, expertise and support and to Julia Blitz for her critical review.

Funding This work was supported by two National Research Foundation Thuthuka grants (TTK2006052200003 and TTK2005072900031) and two Harry and Doris Crossley awards from Stellenbosch University. 
Competing interests None.

Patient consent Informed written consent was obtained.

Ethics approval Ethics approval was given by the Health Research Ethics Committee at Stellenbosch University (reference no 6/10/216).

Contributors Conception and design: KJ and RM. Data collection: KJ and research assistants. Analysis of the data: KJ, M Kidd (statistician). Interpretation of the data: KJ and RM. Drafting of the article: KJ and RM. Critical revision of the article for important intellectual content: KJ, RM and $\mathrm{J}$ Blitz. Final approval of the article: KJ and RM.

Provenance and peer review Not commissioned; externally peer reviewed.

Data sharing statement http://bmjopen.bmj.

com/site/about/resources/datamanagement.xhtml. Informed consent form - to be requested from authors should it be required. Appendix A-first interview tool, previously uploaded. Appendix B-follow-up interview tool, previously uploaded.

\section{REFERENCES}

1. Norman R, Bradshaw D, Schneider M, et al. Estimating the burden of disease attributable to interpersonal violence in South Africa in 2000. $S$ Afr Med J 2007;97:653-6.

2. Abrahams N, Jewkes R, Martin LJ, et al. Mortality of women from intimate partner violence in South Africa: a national epidemiological study. Violence Vict 2009;24:546-56.

3. De Villiers P. Home Violence in South Africa: Surveillance During 1996 by SASPREN, a Sentinel Network of General/Family Practitioners. Cape Town: SASPREN News, 1998 September/ October:87-9.

4. Ratele K. Masculinity and male mortality in South Africa. African Safety Promotion 2008;6:19-41.

5. Gouws A. Unthinking Citizenship. Cape Town: UCT Press, 2005.

6. Domestic Violence Act 116 of 1998 [Online] [s.a.]. http://www.info gov.za/view/DynamicAction?pageid $=545 \&$ sdate $=\%$ 201998\&orderby=act_no\%20desc (accessed 11 Jul 2011).

7. Jewkes R. Reflections on gender violence in the South African public health agenda. Development 2001;44:64-8.

8. Garcia-Moreno C, Jansen HAFM, Ellsberg M, et al. World Health Organization's Multi-Country Study on Women's Health and Domestic Violence: Initial Results on Prevalence, Health Outcomes and Women's Responses. Geneva: World Health Organization Press, 2005.

9. McFarlane JM, Goff JY, O'Brien JA, et al. Secondary prevention of intimate partner violence: a randomized controlled trial. Nurs Research 2006;55:52-61.

10. Wathen $\mathrm{CN}$, MacMillan $\mathrm{HL}$. Interventions for violence against women: scientific review. JAMA 2003;289:589-600.

11. Ramsay J, Richardson J, Carter YH, et al. Should health professional screen women for domestic violence? Systematic review. BMJ 2002;325:1-13;1419.

12. Cherniak $\mathrm{D}$, Grant $\mathrm{L}$, Mason $\mathrm{R}$, et al. Intimate partner violence consensus statement (In English). J Obstet Gynaecol Can 2005;27:365-418.
13. Joyner K, Mash B. Recognizing intimate partner violence in primary care: western cape, South Africa. PLOS ONE. (In press).

14. Joyner K. Health Care for Intimate Partner Violence: Current Standard of Care and Development of Protocol Management. [Dissertation]. Stellenbosch: Stellenbosch University, 2009. http://hdl. handle.net/10019.1/2515 (accessed 31 Aug 2011).

15. Janssen $\mathrm{P}$, Daschal-Weichhendler $\mathrm{H}, \mathrm{McG}$ regor $\mathrm{M}$. Assessment for intimate partner violence: where do we stand? J Am Board Fam Med 2006;19:413-15.

16. Jewkes R. Preventing domestic violence. BMJ 2002;324:253-4.

17. Bradley F, Smith M, Long J, et al. Reported frequency of domestic violence: cross sectional survey of women attending general practice. BMJ 2002;324:274-8.

18. Martin LJ, Jacobs T. Screening for Domestic Violence: a Policy and Management Framework for the Health Sector. Cape Town: Institute of Criminology, University of Cape Town, 2003.

19. Global Programme on Evidence for Health Policy. Putting Women's Safety First: Ethical and Safety Recommendations for Research on Domestic Violence Against Women. Geneva: World Health Organization, 1999. http://whqlibdoc.who.int/hq/1999/ WHO_EIP_GPE_99.2.pdf (accessed 2 Sep 2011).

20. Mash B. How to design education on mental disorders for general practitioners in South Africa. S Afr Fam Pract 2002;25:4-10.

21. Ritchie J, Spencer L. Qualitative data analysis for applied policy research. In: Bryman A, Burgess R, eds. Analysing Qualitative Data. London: Routledge, 1993:173-94.

22. Mathews S, Abrahams N. Combining Stories and Numbers: an Analysis of the Impact of the Domestic Violence Act (No. 116 of 1998) on Women. Tygerberg: The Gender Advocacy Programme and the Medical Research Council, 2001.

23. Limandri B. The therapeutic relationship with abused women: nurses responses that facilitate or inhibit change. J Psychosoc Nurs Ment Health Serv 1987;25:9-16.

24. Bauer HM, Rodriguez MA. Letting compassion open the door: battered women's disclosure to medical providers. Camb Q Healthc Ethics 1995:4:459-65.

25. Chescheir N. Violence against women: response from clinicians. Ann Emerg Med 1996;27:766-8.

26. Rhodes KV, Frankel RM, Levinthal N, et al. "You're not a victim of domestic violence, are you?" Provider-patient communication about domestic violence. Ann Intern Med 2007;147:620-7.

27. Duxbury F. Recognising domestic violence in clinical practice using the diagnoses of postraumatic stress disorder, depression and low self-esteem. Br J Gen Pract 2006;56:294-300.

28. Stenius VM, Veysey BM. "It's the little things": women, trauma and strategies for healing. $J$ Interpers Violence 2005;20:1155-74.

29. Hathaway JE, Zimmer B, Willis G, et al. Perceived changes in health and safety following participation in a health care-based domestic violence program. J Midwifery Womens Health 2008;53:547-55.

30. Joyner K, Mash B. How to provide comprehensive, appropriate care for survivors of intimate partner violence. In: Joyner K, ed. Aspects of Forensic Medicine: an Introduction for Healthcare Professionals. Cape Town: Juta 2010:87-100.

31. Rollnick S, Miller WR, Butler CC. Motivational Interviewing in Health Care: Helping Patients Change Behavior. New York: The Guilford Press, 2008. 


\section{Correction}

Joyner K, Mash RJ. The value of intervening for intimate partner violence in South African primary care: project evaluation. BMJ Open 2011;1:e000254.

In the first key message of the article summary box "listening" was inadvertently omitted. The sentence should read: "Women diagnosed with IPV in primary care perceive benefit from an intervention characterised by both empathic, non-judgemental listening and a comprehensive approach to the clinical, mental, social and legal aspects." The journal apologises for this error.

BMJ Open 2012;2:e00254corr1. doi:10.1136/bmjopen-2011-000254corr1 